\title{
A rare postoperative complication of anterior lower thoracic instrumentation: diaphragmatic laceration with hemothorax
}

1

\section{Abstract}

3 Study Design: Case Report

4 Objective: To highlight the importance of preventing visceral injury due to prominent

5 anterior implants to the spinal column.

6 Summary of Background Data: Spinal cord decompression through a thoracotomy

7 approach is a commonly performed procedure. Postoperative hemothorax is a rare

8 complication but if it occurs, requires urgent attention and reoperation.

9 Methods: A 52-year-old man with cord compression was treated with a T9/10 discectomy

10 and instrumented fusion via a right thoracotomy and trans-pleural approach. Postoperatively

11 he had improved lower limb numbness. However, after a bout of coughing, there was sudden

12 increase in chest drain output, hemodynamic instability and massive fluid collection in the

13 right chest cavity.

14 Results: Emergency re-exploration of the thoracotomy was performed and a $6 \mathrm{~cm}$ laceration

15 of the right postero-medial diaphragm was identified as the bleeding source and was in close 
1 proximity with the locking nut of the anterior implants. The laceration was repaired and a soft

2 synthetic patch was used to cover the implants. Postoperatively, the hemothorax resolved and

3 the patient recovered from the neurological deficit.

4 Conclusions: Prevention of diaphragmatic injury can be performed by using lower profile

5 and less sharp-edged implants or covering the implants with a soft synthetic material if

6 unable to perform direct repair of the parietal pleura.

7

8 Key Words: Hemothorax; anterior spinal fusion; diaphragm; parietal pleura

9

10 Key Points:

11 1. Hemothorax may occur as a result of diaphragmatic laceration after anterior spinal fusion.

2. Low-profile implants should be used in anterior instrumentation.

3. Good coverage of implants with the parietal pleura is advised and a soft synthetic patch should be used to cover implants in case the parietal pleura coverage is inadequate.

Mini Abstract: 
1 prominent and sharp anterior spinal instrumentation which required re-exploration, repair and

2 soft synthetic patch coverage of the implants. Low-profile implants and good coverage of

3 implants with the parietal pleura is advised for lower thoracic anterior instrumentation.

4

\section{Introduction}

6 Spinal cord decompression by discectomy and instrumented fusion through a

7 thoracotomy approach is a commonly used procedure. ${ }^{1,2}$ Postoperative spontaneous

8 hemothorax is a known but rare complication after this transpleural approach to the thoracic

9 spine. $^{3-5}$ It may be caused by re-bleeding of the epidural vessels, oozing from the exposed

10 bone surfaces, inadvertent lung laceration or vascular injury. These causes are usually

11 identified intraoperatively or in the immediate postoperative period. However, late

12 presentation up to 10 months postoperatively have been reported due to the violation of

13 inferior phrenic artery by the anterior instrumentation. ${ }^{4}$ Delay in diagnosis of early

14 hemothorax is unlikely because of obvious clinical manifestations like high chest drain output,

15 hemodynamic instability and drop in hemoglobin level. A simple chest X-ray (CXR) can

16 easily confirm the diagnosis of hemothorax.

17 We report a case of early spontaneous hemothorax caused by a diaphragmatic laceration

18 from anterior instrumentation. This case report serves the purpose of reminding surgeons to

19 identify and prevent potential visceral injury intraoperatively. This can be done by either 
1 using lower profile and less sharp-edged implants or covering the implants with soft synthetic

2 material if the parietal pleura closure cannot provide adequate coverage of the implants.

3 During exploration of a postoperative hemothorax, meticulous examination of the

4 diaphragmatic surface opposing the implants is necessary to control the possible sources of

5 hemorrhage.

6

\section{Case report}

8 A 52-year-old man with history of thoracolumbar back pain for many years was

9 admitted because of spontaneous onset of gradual lower limb weakness and numbness for

10 three days. The right lower limb was 3/5 by Medical Research Council grading (MRC

11 grading) whereas the left side remained 5/5. Sensory level was at T11 and the sensation in

12 both lower limbs was affected. He also had unsustainable ankle clonus and up-going Babinski

13 sign in the right lower limb. He did not have sphincter dysfunction and his anal tone was

14 intact. X-ray examination of the thoracic and lumbar spine was unremarkable. MRI revealed

15 T9/10 prolapsed intervertebral disc causing spinal cord compression with myelomalacic

16 changes (Figures 1a and 1b).

17 Urgent anterior T9/10 discectomy and instrumented fusion was performed through a

18 right T8 thoracotomy and trans-pleural approach. At the end of the surgery, the implants

19 (Universal Spinal System Anterior, Synthes ${ }^{\circledR}$ ) could only be covered partially by the parietal 
1 pleura due to the bulkiness of the implants and no additional mobilization of the parietal

2 pleura. The thoracotomy was then repaired over one chest tube. The patient reported reduced

3 numbness at lower limbs immediately after the surgery. No hemothorax was seen on plain

4 chest radiograph CXR 6 hours after the surgery (Figures 2a and 2b) and the chest drain

5 output was only $100 \mathrm{ml}$. He was hemodynamically stable at the time.

6 The patient subsequently coughed vigorously due to sputum retention and reported

7 immediate retrosternal pain. Thereafter, the chest drain output increased to $1200 \mathrm{ml}$ in 8 hours

8 and his blood pressure became labile. Repeat CXR 14 hours after the initial operation showed

9 significant fluid accumulation inside the right thorax (Figure 2c). Together with the rapidity

10 of onset following bouts of heavy coughing, hemothorax was highly suspicious. He was

11 immediately taken back to the operation theater for re-exploration of the right thoracotomy.

12 While re-opening the thoracotomy, the patient had hiccoughs despite of general anesthesia. A

13 right postero-medial diaphragmatic laceration of about $6 \mathrm{~cm}$ opposing the spinal implant was

14 found with active bleeding (Figures $3 \mathbf{a}$ and $\mathbf{3 b}$ ). The laceration was repaired and the spinal

15 implants were covered by a synthetic dural patch (Neuro-Patch ${ }^{\circledR}$, Aesculap Inc.) to prevent

16 any further laceration of the diaphragm (Figure 3c). Old blood clots were removed from the

17 pleural cavity and the thoracotomy was repaired over two chest tubes.

The subsequent chest drain output was not excessive and the chest tubes were removed 
1 recurrent hemothorax and the postoperative thoracic spine $\mathrm{x}$-ray demonstrated satisfactory

2 implant position (Figures $\mathbf{4 a}$ and $\mathbf{4 b}$ ). The patient had near-full neurologic function two

3 weeks after the spinal cord decompression and was able to walk independently without any

4 aids.

5

6 Discussion

7 The diaphragm is a dome-shaped muscle that is important for respiration. During

8 inspiration, the muscle contracts and the dome flattens. As a result, the intra-thoracic volume

9 is increased and the intra-thoracic pressure is decreased with passive air entry into the lungs.

10 During expiration, the diaphragmatic muscles relax and the dome shape is resumed. This

11 increases the intra-thoracic pressure and air is expelled from the chest cavity. When the

12 diaphragm is in its dome shape, the peripheral muscular part is in close proximity with the

13 lower chest wall and the lower thoracic spine. During the coughing effort, the intra-thoracic

14 pressure is greatly increased by the contraction of the abdominal muscles. ${ }^{6}$ This increase in

15 intra-abdominal pressure causes a rigorous upward thrusting of the diaphragm which expels

16 the air out of the lungs in an effort to cough up sputum. Such violent upward thrust of the

17 diaphragm induces a strong gliding movement between the lower thoracic spine and the

18 diaphragm. Any hard prominent object in its path will impede this smooth gliding motion and may even lacerate the diaphragm. Both hemi-diaphragms move across a few intercostal 
1 spaces within a single breathing cycle. The right hemi-diaphragm usually lies on a more

2 rostral position due to the underlying liver. In addition, there are regional differences in the

3 movement of the right hemi-diaphragm. The middle and posterior thirds of the right

4 hemi-diaphragm have greater excursion than the anterior third. ${ }^{7}$ As a result, the posterior part

5 of the right hemi-diaphragm is prone to injury by prominent implants inserted in the lower

6 thoracic spine.

Under normal circumstances, gliding movement within the pleural cavity has minimal

8 friction due to the smooth surfaces between the parietal and visceral pleura as well as the

9 lubricating pleural fluid. ${ }^{8}$ Although it is desirable to repair the parietal pleura to provide a

10 barrier between the implants and the pleural cavity, it is not always feasible due to the

11 bulkiness of the implants and relative inelasticity of the pleura. Possible solutions include

12 mobilizing a larger area of parietal pleura to close the pleural cavity over the implants or use

13 a smooth synthetic patch to cover the implants. This avoids violation of the nearby lung or

14 diaphragm by the edge of the implants. Furthermore, lower profile implants facilitate

15 complete closure of the parietal pleura.

16 Postoperative diaphragmatic laceration due to anterior lower thoracic spinal

17 instrumentation has not been reported previously. Theoretically, instrumentation on the right

18 side with chest tube insertion may be additional risk factors for the scenario presented in our

case. On the right side, the liver may push the right hemi-diaphragm hard against the 
1 prominence of the lower thoracic implants. Hence, a left-sided approach may be safer.

2 However, since the weakness of the patient was on the right side, right sided approach offered

3 direct decompression. In addition, chest tube insertion often induces considerable pain during

4 breathing. To minimize such pain, patients may use more diaphragmatic breathing and the

5 diaphragm may rub again the implants more. Whether this postulation is true and makes

6 patients more susceptible to diaphragmatic injury requires further study.

7 It is difficult to be completely sure whether the diaphragmatic laceration was not caused

8 by other reasons such as intraoperative inadvertent injury. However, the circumstantial

9 evidence and timing of the hemothorax supports our postulation. The early CXR did not

10 reveal any hemothorax and the chest drain was minimal after surgery. Yet, subsequent to the

11 patient's episode of vigorous cough and retrosternal pain, the hemodynamics became labile,

12 chest drain output increased significantly and a massive hemothorax was detected in the

13 following CXR. Furthermore, hiccough appeared during the re-exploration which indicates

14 diaphragmatic irritation.

16 Conclusion

17 Surgeons should select low profile implants at the lower thoracic spine for the anterior

18 instrumentation. Complete coverage of implants by repair of the parietal pleura is desirable.

19 In case of incomplete coverage due to tight or friable parietal pleura, surgeons should 
1 consider using a smooth synthetic substance to cover the implants. A left sided approach may

2 be safer but the choice of approaching sides may be dictated by the pathology.

3 
1

2 1. Ayhan S, Nelson C, Gok B, et al. Transthoracic surgical treatment for centrally located

\section{References} thoracic disc herniations presenting with myelopathy: a 5-year institutional experience.

4 Journal of spinal disorders \& techniques 2010;23:79-88.

5

6

7 3. Coe JD, Arlet V, Donaldson W, et al. Complications in spinal fusion for adolescent

2. Quraishi NA, Khurana A, Tsegaye MM, et al. Calcified giant thoracic disc herniations: considerations and treatment strategies. Eur Spine J 2014;23 Suppl 1:S76-83.

idiopathic scoliosis in the new millennium. A report of the Scoliosis Research Society Morbidity and Mortality Committee. Spine (Phila Pa 1976) 2006;31:345-9.

4. Geervliet PC, van Royen BJ, Vonk Noordegraaf A, et al. Late spontaneous hemothorax complicating anterior spinal instrumentation in adolescent idiopathic scoliosis. Spine (Phila Pa 1976) 2007;32:E730-3.

5. Shapiro G, Green DW, Fatica NS, et al. Medical complications in scoliosis surgery. Curr Opin Pediatr 2001;13:36-41.

6. Pennock BE. Descriptive ventilatory mechanics from noninvasive analog display of ribcage and abdominal flow. Ann Biomed Eng 1992;20:559-72.

7. Harris RS, Giovannetti M, Kim BK. Normal ventilatory movement of the right hemidiaphragm studied by ultrasonography and pneumotachography. Radiology 1983;146:141-4. 
1 8. Finley DJ, Rusch VW. Anatomy of the pleura. Thorac Surg Clin 2011;21:157-63, vii.

\section{Figure Legends}

4 Figure 1: Preoperative a) sagittal and b) axial T2-weighted MRI showing a huge T9/10

5 prolapsed intervertebral disc causing spinal cord compression and myelomalacic changes of

6 the spinal cord.

7 Figure 2: Chest x-ray taken a) preoperatively was clear. Subsequent x-rays taken after the

8 T9/10 anterior decompression and instrumented fusion at b) 6 hours postoperatively showed

9 no hemothorax but a massive right hemothorax was observed at c) 14 hours postoperatively.

10 Figure 3: Intraoperative photos of the reoperation showed a) the diaphragmatic laceration

11 opposite the locking nut of the screw. The laceration was repaired and b) it was identified to

12 be in close proximity of the sharp edge of the locking nut. c) A synthetic material was used to

13 provide adequate coverage the sharp locking nut. 